\title{
OPEN Viability fingerprint of glioblastoma cell lines: roles of mitotic, proliferative, and epigenetic targets
}

\author{
Darja Lavogina ${ }^{1,2 \bowtie}$, Tõnis Laasfeld ${ }^{2,3}$, Markus Vardja ${ }^{4}$, Helen Lust ${ }^{1} \&$ Jana Jaal ${ }^{1,4} \bowtie$
}

Despite the use of multimodal treatment combinations, the prognosis of glioblastoma (GB) is still poor. To prevent rapid tumor recurrence, targeted strategies for the treatment of $G B$ are widely sought. Here, we compared the efficacy of focused modulation of a set of signaling pathways in two GB cell lines, U-251 MG and T98-G, using a panel of thirteen compounds targeting cell cycle progression, proliferation, epigenetic modifications, and DNA repair mechanism. In parallel, we tested combinations of these compounds with temozolomide and lomustine, the standard chemotherapy agents used in GB treatment. Two major trends were found: within individual compounds, the lowest IC $C_{50}$ values were exhibited by the Aurora kinase inhibitors, whereas in the case of mixtures, the addition of DNA methyltransferase 1 inhibitor azacytidine to lomustine proved the most beneficial. The efficacy of cell cycle-targeting compounds was further augmented by combination with radiation therapy using two different treatment regimes. The potency of azacytidine and lomustine mixtures was validated using a unique assay pipeline that utilizes automated imaging and machine learning-based data analysis algorithm for assessment of cell number and DNA damage extent. Based on our results, the combination of azacytidine and lomustine should be tested in GB clinical trials.

\author{
Abbreviations \\ DNMT DNA methyltransferase \\ GB Glioblastoma \\ SAHA Suberanilohydroxamic acid
}

Glioblastoma (GB) is the most aggressive and lethal brain cancer in adults. Despite the use of multimodal treatment combinations, the prognosis of GB is still poor ${ }^{1}$. Since 1978, postoperative radiotherapy has been the mainstay of standard adjuvant treatment in $\mathrm{GB}^{2}$, which results in median overall survival of 12.1 months that can be extended to 14.6 months by administering temozolomide during and after radiotherapy ${ }^{3}$. In patients 70 years of age or younger, additional alternating electric field therapy can be utilized in combination with radiotherapy and temozolomide that prolongs median overall survival up to 20.9 months ${ }^{4}$.

Unfortunately, almost all GB patients die due to rapid tumor recurrence. Currently, no standard of care treatment is established when the disease progresses after radiotherapy and temozolomide. Out of possible treatment options that include repeat surgery and chemotherapy, a nitrosourea compound lomustine is most commonly used at disease recurrence. This approach is based on several phase II and III studies that have reported median overall survival times ranging from 5.6 to 9.8 months $\mathrm{s}^{5-9}$. Moreover, previous studies have shown that re-irradiation provides temporary disease control in recurrent GB patients. A systematic review including 50 non-comparative studies of 2095 patients with recurrent GB who were treated with re-irradiation showed pooled 6- and 12 -month overall survival rates of $73 \%$ and $36 \%{ }^{10}$.

Since chemotherapy and radiotherapy result only in temporary disease stabilization in GB patients, additional, targeted strategies for the treatment of GB are widely sought. Among other options, selective modulation of epigenetic targets (e.g., DNA methyltransferases or histone deacetylases), cell cycle-related targets (e.g., mitotic protein kinases from the Aurora family), targets involved in DNA repair mechanisms and checkpoints

${ }^{1}$ Institute of Clinical Medicine, University of Tartu, L. Puusepa 8, 50406 Tartu, Estonia. ${ }^{2}$ Institute of Chemistry, University of Tartu, Tartu, Estonia. ${ }^{3}$ Department of Computer Science, University of Tartu, Tartu, Estonia. ${ }^{4}$ Department of Radiotherapy and Oncological Therapy, Tartu University Hospital, Tartu, Estonia. ${ }^{\square}$ email: darja.lavogina@ut.ee; jana.jaal@kliinikum.ee 


\begin{tabular}{|c|c|c|c|c|c|c|}
\hline \multirow[b]{2}{*}{ Compound } & \multicolumn{3}{|l|}{ U-251 MG } & \multicolumn{3}{|l|}{ T98-G } \\
\hline & $\mathrm{pIC}_{50}[\mathrm{CI}]$ & $\mathrm{IC}_{50}, \mu \mathrm{M}$ & $\begin{array}{l}\text { Bottom plateau, \% } \\
\text { [CI] }\end{array}$ & $\mathrm{pIC}_{50}[\mathrm{CI}]$ & $\mathrm{IC}_{50}, \mu \mathrm{M}$ & $\begin{array}{l}\text { Bottom plateau, \% } \\
\text { [CI] }\end{array}$ \\
\hline Temozolomide & $3.50[3.63$ to 3.37$]$ & 317 & $0[-5$ to 4$]$ (ns) & $3.60[3.73$ to 3.48$]$ & 249 & $0[-5$ to 5$]$ (ns) \\
\hline Lomustine & $4.50[4.57$ to 4.43$]$ & 31.6 & $0[-3$ to 4$]$ (ns) & $4.08[4.15$ to 4.00$]$ & 84.1 & $0[-5$ to 4$](\mathrm{ns})$ \\
\hline Pasireotide & $4.02[4.28$ to 3.76$]$ & 96.4 & $0[-8$ to 8$]$ (ns) & $4.00[4.30$ to 3.71$]$ & 99.3 & $0[-10$ to 10$](\mathrm{ns})$ \\
\hline CYN 154,806 & $3.02[3.45$ to 2.58$]$ & 962 & $0[-9$ to 8$]$ (ns) & $3.44[3.69$ to 3.20$]$ & 360 & $0[-10$ to 9$]$ (ns) \\
\hline MK-2206 & $5.17[5.28$ to 5.06$]$ & 6.82 & $1[-4$ to 6$](\mathrm{ns})$ & $4.77[4.88$ to 4.66$]$ & 17.1 & $-6[-12$ to 1$]$ (ns) \\
\hline ARQ 092 & $5.06[5.17$ to 4.96$]$ & 8.72 & $-3[-8$ to 3$]$ (ns) & 4.54 [4.67 to 4.42$]$ & 28.9 & $-5[-13$ to 2$](\mathrm{ns})$ \\
\hline ARC-775 & 4.84 [4.96 to 4.73$]$ & 14.3 & 0 [-8 to 9] (ns) & $4.71[4.84$ to 4.57$]$ & 19.7 & $1[-9$ to 10$]$ (ns) \\
\hline CX-4945 & $5.30[5.41$ to 5.19$]$ & 5.05 & $4[-5$ to 11$](\mathrm{ns})$ & $5.17[5.30$ to 5.04$]$ & 6.79 & $3[-7$ to 12$]$ (ns) \\
\hline SAHA & 4.83 [4.94 to 4.73$]$ & 14.8 & $-1[-7$ to 4$]$ (ns) & 4.70 [4.82 to 4.59$]$ & 20.0 & $-4[-11$ to 2$](\mathrm{ns})$ \\
\hline Azacytidine & 5.11 [5.24 to 5.00$]$ & 7.68 & 12 [7 to 17] $\left(^{* * *}\right)$ & $4.99[5.12$ to 4.86$]$ & 10.3 & $6[0$ to 12$](\mathrm{ns})$ \\
\hline MK-1775 & $5.69[5.83$ to 5.55$]$ & 2.06 & $27[23$ to 30$]\left({ }^{* * *}\right)$ & $6.47[6.60$ to 6.34$]$ & 0.341 & $17[14$ to 21$]\left(^{(* *}\right)$ \\
\hline CC-115 & $6.33[6.43$ to 6.24$]$ & 0.469 & $17[14 \text { to } 19]^{(* *)}$ & $5.57[5.69$ to 5.46$]$ & 2.68 & $16[13$ to 20$\left.]{ }^{(* *}\right)$ \\
\hline MLN8237 & 10.0 [10.4 to 9.69$]$ & 0.0000979 & $43[39 \text { to } 48]^{(* *)}$ & 10.2 [10.7 to 9.69$]$ & 0.0000669 & $56[52$ to 61$]\left(^{(* *)}\right.$ \\
\hline VX 689 & 7.22 [7.53 to 6.93$]$ & 0.0597 & $49[43$ to 55$]]^{(* *)}$ & 7.23 [7.59 to 6.87$]$ & 0.0589 & $54[47$ to 61$]\left(^{(* *)}\right.$ \\
\hline AZD1152-HQPA & $7.63[7.97$ to 7.30$]$ & 0.0235 & $66[62$ to 70$]\left({ }^{* * *}\right)$ & $6.00[6.40$ to 5.60$]$ & 1.01 & $67[61$ to 73$]\left({ }^{* * *}\right)$ \\
\hline
\end{tabular}

Table 1. Effect of individual compounds on viability of GBM cell lines ( $\geq 4)$. CI, 95\% confidence interval; ns, not significant; $\mathrm{pIC}_{50}$, negative logarithm of the half maximal inhibitory concentration. The asterisks indicate the significance of grouped comparison of the dose-response bottom plateau value obtained for each treatment condition relative to zero (one-way ANOVA; ${ }^{\star * *} P<0.001$; ns, not significant).

(e.g., DNA-PK and Wee1) and multiple targets involved in proliferative and anti-apoptotic signaling pathways (e.g., somatostatin receptor and protein kinases Akt/PKB and CK2) is actively explored ${ }^{11-13}$. Although there are numerous reports investigating the efficiency of individual compounds, few studies have focused on a systematic comparison of roles of different pathways that define the characteristic viability profile of GB.

Here, we set out to compare the efficacy of targeted modulation of a set of signaling pathways in two GB cell lines, U-251 MG and T98-G. These cell lines differ in O6-methylguanine-DNA methyltransferase (MGMT) protein expression (T98-G is MGMT-positive and U-251 MG is MGMT-negative) and sensitivity to cytotoxic treatments (T98-G has shown high resistance to both radiotherapy and temozolomide ${ }^{14}$ ). By catalyzing demethylation of O6-methylguanine, MGMT contributes to the resistance of the cells to drugs that act via DNA alkylation; up to today, MGMT is the most studied prognostic biomarker in GB patients ${ }^{15}$.

For the assessment of targeted modulation in GB cells, thirteen individual compounds with focused selectivity profiles and combinations thereof with temozolomide and lomustine were screened using viability assay. After establishing the hotspots of interest, we also assessed the efficacy of one targeted drug candidate in combination with irradiation. Moreover, to characterize the effects of tested compounds and their combinations, fluorescence microscopy in fixed cells combined with the machine learning-based image processing pipeline was used.

\section{Results}

Viability assay. For establishing the viability fingerprint via modulation of the chosen signaling pathways, we defined a set of compounds of interest based on the relevant biological targets (see Supplementary Table S1). The candidates were chosen based on the available data regarding their potency, selectivity, and blood-brain barrier permeability; whenever possible, drugs that are currently used in clinics, and drug candidates that have advanced to the later stages of clinical trials were preferred ${ }^{16-31}$. The only compounds that have not been tested in clinical trials were somatostatin receptor (SSTR) antagonist CYN $154806^{32,33}$, and casein kinase 2 (CK2) inhibitor ARC- $775^{34}$.

The dose-response effect of individual compounds on viability of U-251 MG and T98-G cell lines following $48 \mathrm{~h}$ treatment is summarized in Table 1 and Fig. 1 . Briefly, while temozolomide and lomustine showed moderate $\mathrm{IC}_{50}$ values in the submillimolar range, several targeted compounds featured remarkably lower $\mathrm{IC}_{50}$ values. Most of the targeted compounds were slightly more active in U-251 MG cells, except for SSTR antagonist CYN 154806 and Wee1 inhibitor MK-1775 for which lower $\mathrm{IC}_{50}$ values were observed in the T98-G line. Among the biological targets of interest, the most pronounced effect was observed in the case of mitotic Aurora kinases, as inhibitors of Aurora A/B featured submicromolar to subnanomolar efficiency in both cell lines. On the other hand, as indicated by the elevated bottom plateau of the dose-response curves (Table 1), these compounds could not trigger full mortality of cell population even at elevated concentrations, but rather abolished the proliferation of cells.

We next analyzed how the viability of GB cell lines was affected by mixtures composed of a targeted compound and a chemotherapy drug (either temozolomide or lomustine). The composition of mixtures was chosen based on the solubility of compounds in water, the comparability of the individual toxicities of compounds to those of temozolomide and lomustine, and the amount of available material. The mixtures tested and the corresponding dose-response $\mathrm{pIC}_{50}$ values are listed in Fig. 2 and Supplementary Figure S1. Briefly, in either cell line, several mixtures proved more efficient than the chemotherapy drugs alone, yet only a few mixtures were significantly more potent than the corresponding individual targeted compound included in the mixture $(P<0.05)$. Among 


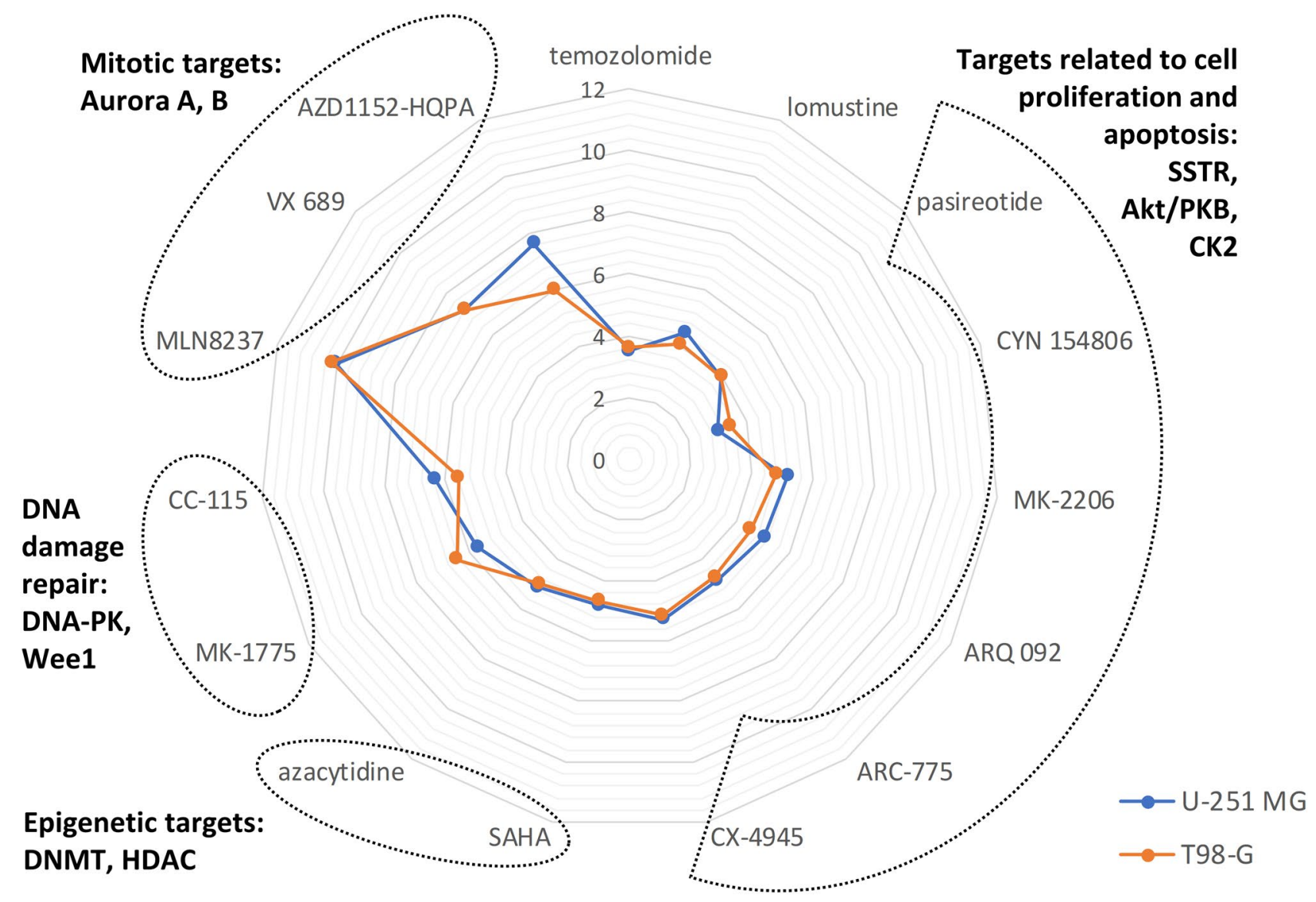

Figure 1. Viability fingerprint of GB cell lines following $48 \mathrm{~h}$ treatment. $\mathrm{pIC}_{50}$ values of individual compounds are shown for U-251 MG (blue) and T98-G (orange) cell lines. The further is the data point located from the center of the plot, the more efficient is the corresponding compound. The major biological targets of compounds are listed on the periphery.

the latter, the most outstanding were 1:1 molar mixtures of azacytidine with chemotherapy drugs, which showed consistently increased efficiency compared to the efficiencies of the corresponding single components. The shift of the dose-response curve in the case of azacytidine + lomustine (1:1) mixture is illustrated in Supplementary Figure S2.

Radiation studies with MLN8237. To confirm the efficiency of Aurora A inhibitors that we observed in the viability studies, we proceeded to combined treatment of cell lines with MLN8237 and radiation (2 Gy or

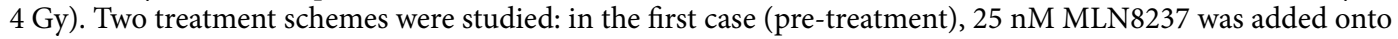
the cells $24 \mathrm{~h}$ prior to the irradiation, whereas in the second case (post-treatment), $25 \mathrm{nM}$ MLN8237 was added onto the cells $2 \mathrm{~h}$ after the irradiation. In both cases, the viability of cells was analyzed $48 \mathrm{~h}$ after irradiation. As the controls, we also tested the effect of treatment with MLN8237 without irradiation, and the effect of irradiation without MLN8237 treatment.

The results of radiation studies are summarized in Fig. 3 and Supplementary Figure S3. Briefly, within the timeframe of the assay, neither 2 Gy nor 4 Gy radiation alone caused a significant reduction of viability in GB cell lines relative to the non-irradiated control. Contrarily, treatment with MLN8237 caused significant reduction in cell viability $(P<0.01)$ in both cell lines for irradiated as well as non-irradiated plates, whereas the pre-treatment was consistently more efficient than the post-treatment $(P<0.05)$. The comparisons within the same treatment scheme revealed that in U-251 MG cells, co-treatment with MLN8237 and 4 Gy radiation was significantly more efficient than MLN8237 alone irrespective of the treatment scheme $(P<0.01)$; in T98-G cells, the combination of 2 Gy irradiation with MLN8237 post-treatment was more efficient than MLN8237 treatment alone according to the $\mathrm{t}$-test $(P<0.05)$, but not according to the Mann-Whitney U-test $(P=0.07$; Supplementary Figure $\mathrm{S} 3)$.

Immunofluorescence (IF) studies. To confirm the enhanced efficiency of lomustine + azacytidine mixture by an alternative methodology, we designed an assay enabling direct counting of cell number following treatment with individual compounds or their mixture. After $48 \mathrm{~h}$ treatment, the cells were fixed and stained with 4,6-diamidino-2-phenylindole (DAPI) to visualize nuclei; in parallel, to assess the DNA damaging effect of compounds and their mixture, immunofluorescent staining with antibody against phosphorylated Ser139 of histone $\mathrm{H} 2 \mathrm{AX}(\gamma \mathrm{H} 2 \mathrm{AX})$ was carried out. For precise quantification of imaging data, an automated data analysis 


\section{Combinations with lomustine: U-251 MG}

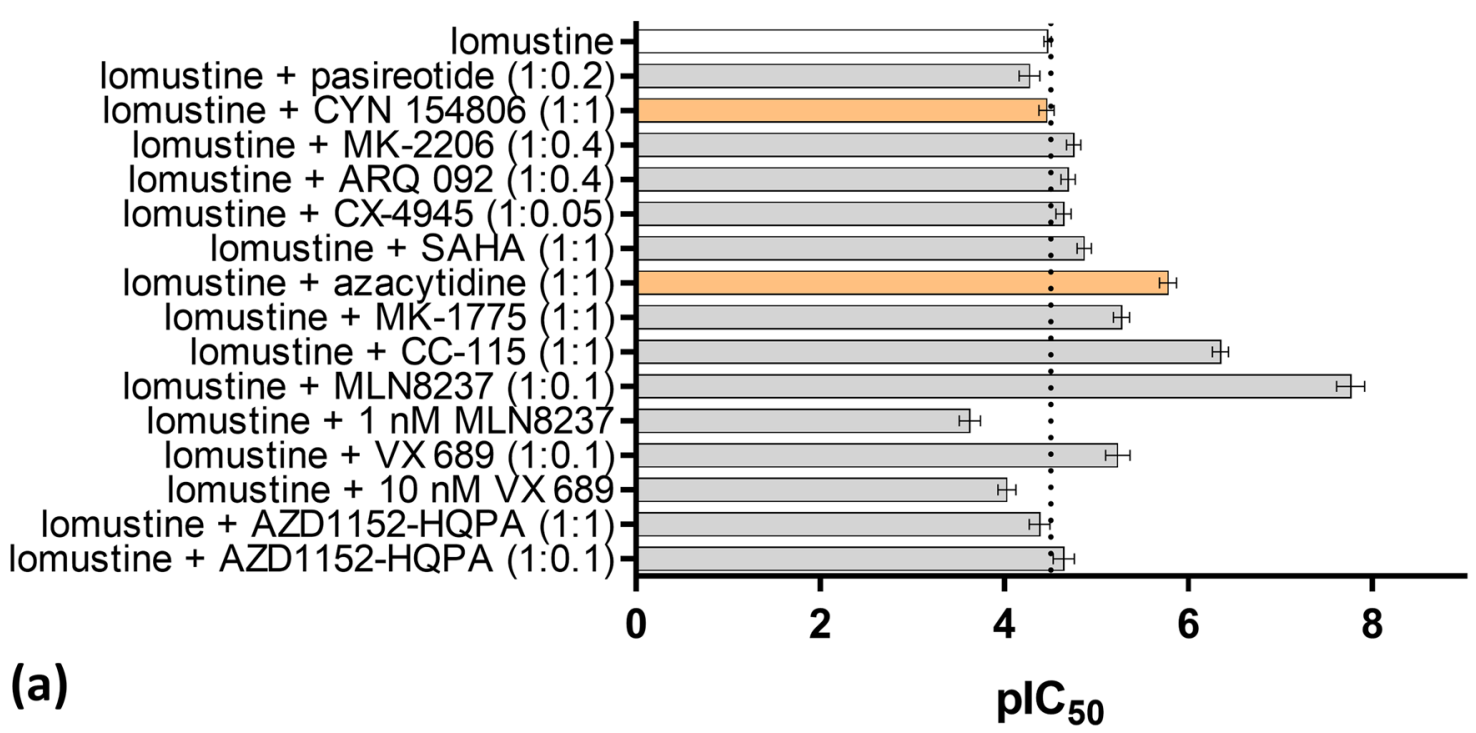

\section{Combinations with lomustine: T98-G}

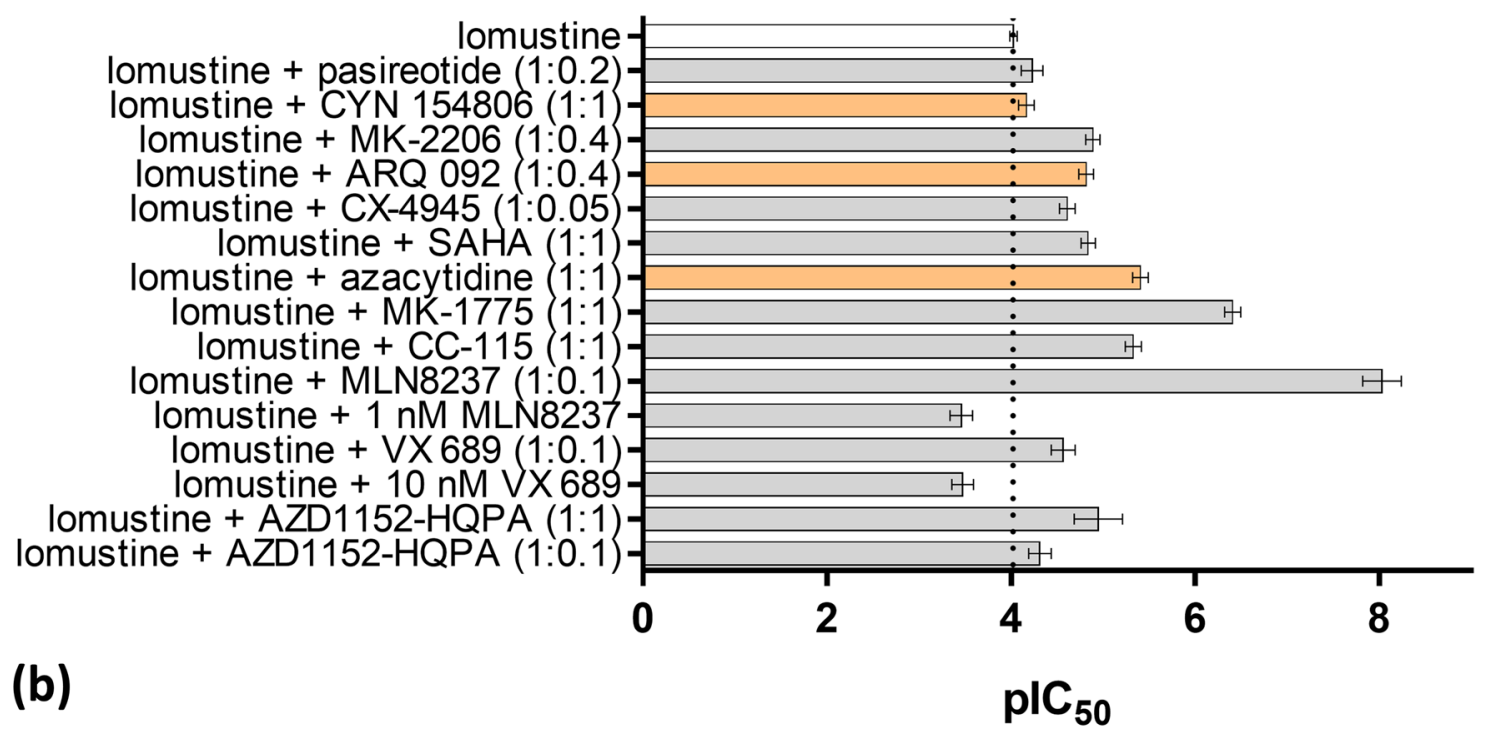

Figure 2. Effect of compound mixtures containing lomustine on the viability of GB cell lines. pIC 50 values \pm standard errors of mean are shown $(\mathrm{N} \geq 4)$ for $\mathrm{U}-251 \mathrm{MG}(\mathbf{a})$ or $\mathrm{T} 98-\mathrm{G}$ (b) following $48 \mathrm{~h}$ treatment. The further each column protrudes to the right, the more efficient is the corresponding mixture. In brackets, the molar ratios of components in each point of the dose-response curve are listed. The dotted vertical line shows the $\mathrm{pIC}_{50}$ value for lomustine alone. The columns colored in orange indicate mixtures for which the $\mathrm{pIC}_{50}$ value was significantly higher than the $\mathrm{pIC}_{50}$ value of either component within the given mixture $(P<0.05)$.

algorithm was developed that utilizes machine learning for detection of nuclei in the obtained images. The nuclei masks are then transferred to the $\gamma \mathrm{H} 2 \mathrm{AX}$ channel to establish the average intensity of antibody staining in each nucleus.

The results of IF studies are summarized in Fig. 4 and Supplementary Figures S4-S5, and the representative microscopy images shown in Fig. 5 and Supplementary Figures S6-S7. The first set of experiments was carried out in both GB cell lines; for straightforward interpretation of the results, we utilized a constant concentration of azacytidine $\left(1 \mu \mathrm{M}\right.$, being thus 8 -tenfold below the $\mathrm{IC}_{50}$ value of the individual compound according to viability studies) and dilution series of lomustine (sixfold dilutions starting from $100 \mu \mathrm{M}$ concentration, in accordance with viability assay setup). Under these conditions, the number of nuclei that could be identified for the $100 \mu \mathrm{M}$ lomustine ( $\pm 1 \mu \mathrm{M}$ azacytidine) treatment was very low (Supplementary Figure S5). However, for the next dilution point, the total number of nuclei per well was sufficient to pool the data for 3 independent experiments (Fig. 5); 
Radiation experiments: U-251 MG

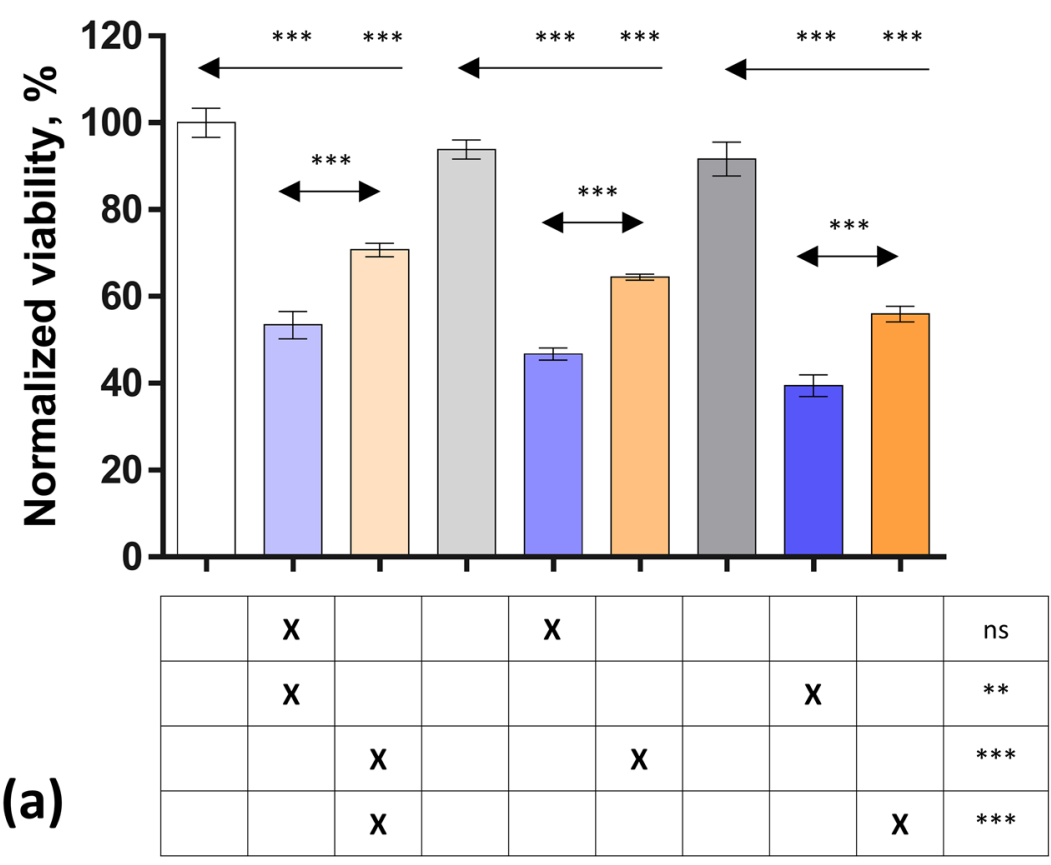

PBS control / no irr

MLN8237 pre-treatment / no irr

MLN8237 post-treatment / no irr

PBS control / 2 Gy

MLN8237 pre-treatment / 2 Gy

MLN8237 post-treatment / 2 Gy

PBS control / 4 Gy

MLN8237 pre-treatment / 4 Gy

MLN8237 post-treatment / 4 Gy

Statistical significance

of pairwise comparisons

(columns indicated by $\mathbf{x}$ )

\section{Radiation experiments: T98-G}
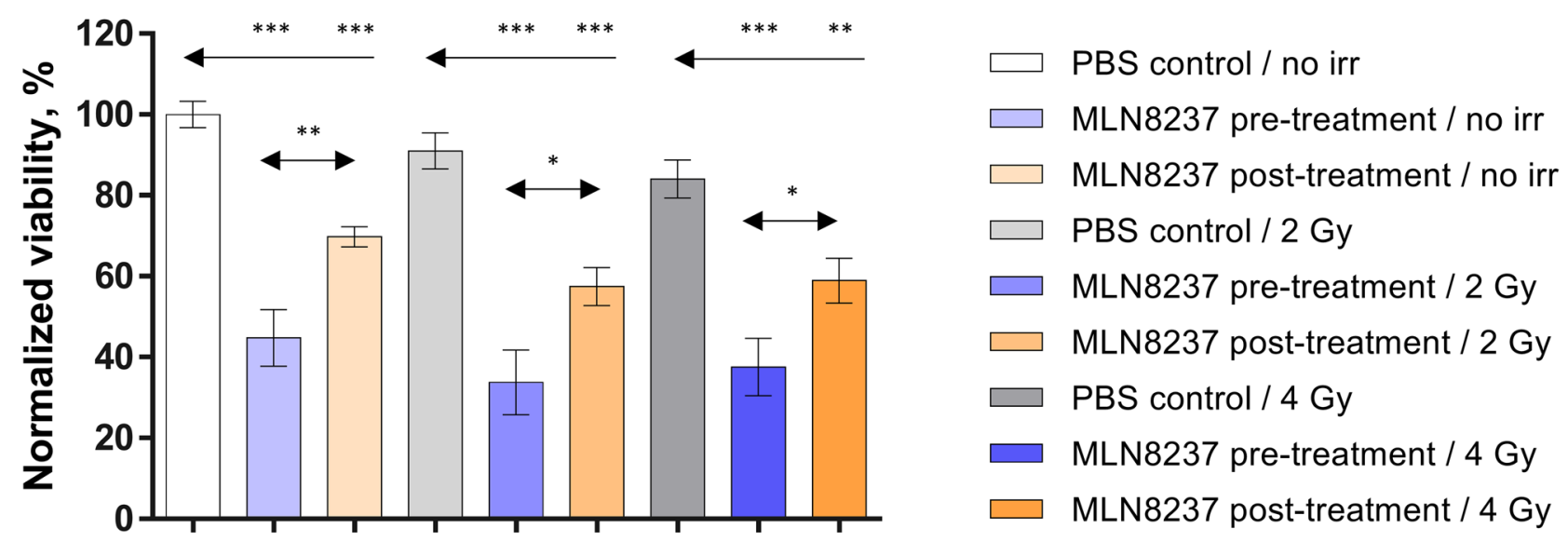

(b)

\begin{tabular}{|c|c|c|c|c|c|c|c|c|c|}
\hline & $\mathbf{X}$ & & & $\mathbf{X}$ & & & & & ns \\
\hline & $\mathbf{X}$ & & & & & & $\mathbf{X}$ & & ns \\
\hline & & $\mathbf{X}$ & & & $\mathbf{X}$ & & & & $*$ \\
\hline & & $\mathbf{X}$ & & & & & & $\mathbf{X}$ & ns \\
\hline
\end{tabular}

\section{Statistical significance of pairwise comparisons (columns indicated by $\mathbf{x}$ )}

Figure 3. Potentiation of irradiation effect by MLN8237. Normalized viability values \pm standard errors of mean are shown $(\mathrm{N}=4)$. MLN8237 (final concentration of $25 \mathrm{nM}$ ) was added onto U-251 MG (a) or T98-G (b) cells either $24 \mathrm{~h}$ prior to irradiation (pre-treatment) or $2 \mathrm{~h}$ following the irradiation (post-treatment). Following irradiation, the cells were grown for a further $48 \mathrm{~h}$. The asterisks above the top arrows in the graphs indicate the significance of grouped comparisons of each treatment condition to the PBS-treated cells on the same plate (one-way ANOVA; ${ }^{* *} P<0.001{ }^{* *} P<0.01$ ). The asterisks above the middle set of arrows in the graphs indicate pairwise comparisons for the pre-treated and post-treated columns (unpaired t-test; ${ }^{* *} P<0.001 ;{ }^{* *} P<0.01$; ${ }^{*}$ $P<0.05$; ns, not significant). The grids below the graphs show pairwise comparisons (the compared columns are indicated with X) for the pre-treated or post-treated cells under different irradiation conditions (unpaired t-test; ${ }^{* * *} \mathrm{P}<0.001 ;{ }^{* *} \mathrm{P}<0.01 ;{ }^{*} \mathrm{P}<0.05 ;$ ns, not significant). 


\section{Cell count per well}

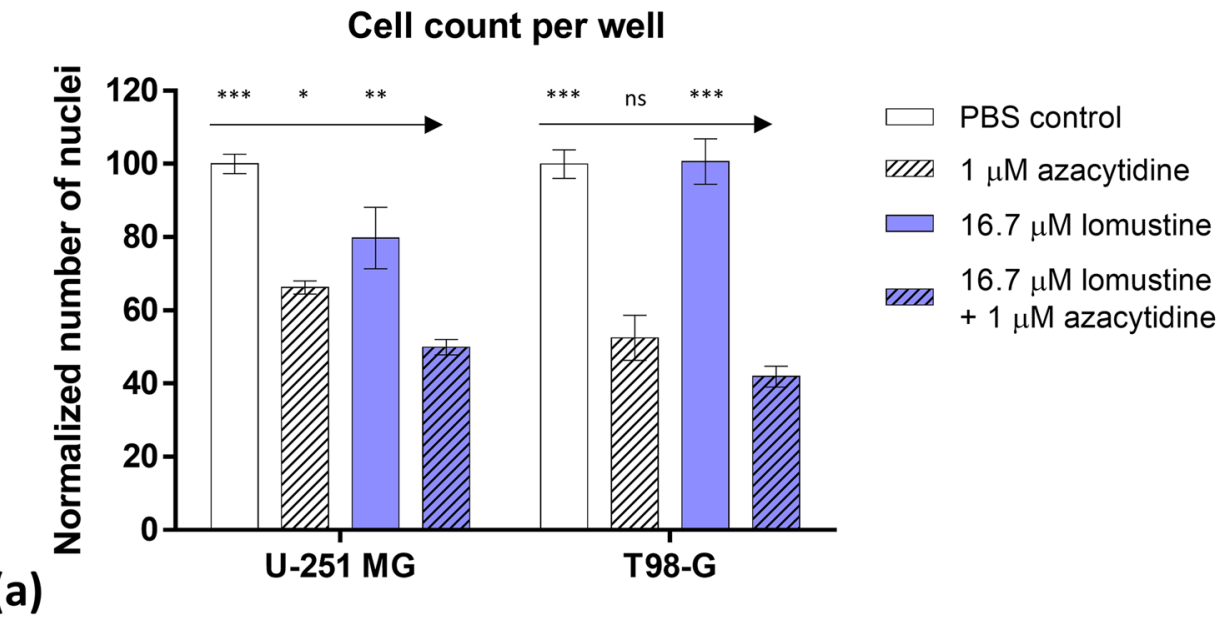

(a)

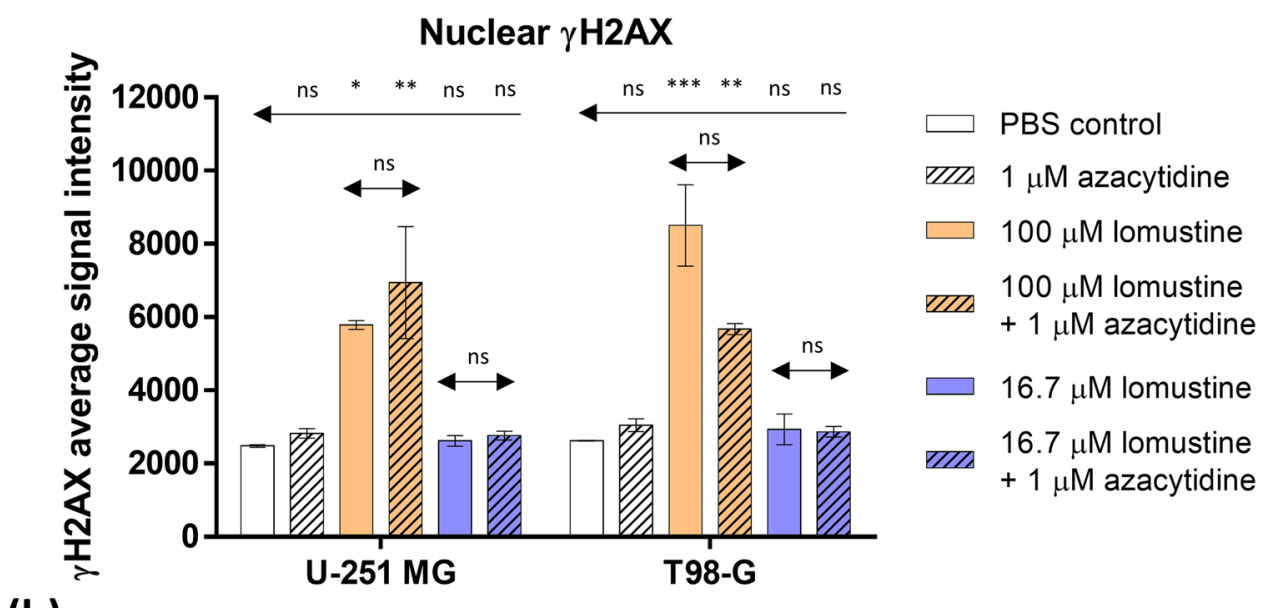

(b)

Figure 4. Confirmation of synergistic effect of lomustine and azacytidine in GB cell lines as determined by IF assay. (a) Effect of individual compounds and their mixture on the cell number following $48 \mathrm{~h}$ treatment. The graph shows the number of nuclei normalized to the PBS control for each individual experiment \pm standard errors of mean $(\mathrm{N}=3)$. The asterisks above the arrows in the graphs indicate the significance of grouped comparison of each treatment condition to the mixture-treated cells in the same cell line (one-way ANOVA; ${ }^{* * *} P<0.001$; ${ }^{* *} P<0.01$; ${ }^{*} P<0.05$; ns, not significant). (b) Effect of individual compounds and their mixtures on the $\gamma \mathrm{H} 2 \mathrm{AX}$ levels in the nucleus following $48 \mathrm{~h}$ treatment. The graph shows mean average $\gamma \mathrm{H} 2 \mathrm{AX}$ signal intensities \pm standard errors of mean $(\mathrm{N}=3)$. The asterisks above the arrows in the graphs indicate the significance of grouped comparison of each treatment condition to the PBS control in the same cell line (one-way ANOVA; ${ }^{* *} P<0.001 ;{ }^{* *} P<0.01 ;{ }^{*} P<0.05$; ns, not significant). The asterisks above the middle set of arrows in the graphs indicate pairwise comparisons for treatments with lomustine $v s$ mixture (unpaired t-test; ns, not significant).

it can be seen from Fig. 4a that the percentage of nuclei [relative to phosphate-buffered saline (PBS) treatment] was significantly higher in U-251 MG cells treated with $16.7 \mu \mathrm{M}$ lomustine or $1 \mu \mathrm{M}$ azacytidine alone, as compared to the corresponding mixture $(P \leq 0.05)$. In T98-G cells, the mixture was significantly more potent than lomustine alone $(P \leq 0.001)$, yet there was no statistically significant difference between the normalized number of nuclei in azacytidine $v s$ mixture treatments.

On the other hand, while in either cell line, the signal intensity of nuclear $\gamma \mathrm{H} 2 \mathrm{AX}$ staining was significantly higher in case of treatment with $100 \mu \mathrm{M}$ lomustine ( $\pm 1 \mu \mathrm{M}$ azacytidine) as compared to the PBS control $(P \leq 0.05)$, there was no significant difference between $100 \mu \mathrm{M}$ lomustine alone and its mixture with azacytidine. At lower concentrations of lomustine, $\gamma \mathrm{H} 2 \mathrm{AX}$ staining was not different from the PBS control (Figs. $4 \mathrm{~b}, 5$ ). The distribution of signals in individual nuclei in different treatments is shown in Supplementary Figure S4.

The second set of experiments $(\mathrm{N}=3)$ was carried out in $\mathrm{U}-251 \mathrm{MG}$ cell line only, and utilized dilution series of lomustine, azacytidine, or 1:1 molar mixture of the compounds (similarly to the viability assay). In this experiment, we calculated the dose-response pIC $_{50}$ values for the effect of individual compounds and their mixture on the cell number. Supplementary Figure S5 shows that the $\mathrm{pIC}_{50}$ values of individual compounds are significantly 
U-251 MG

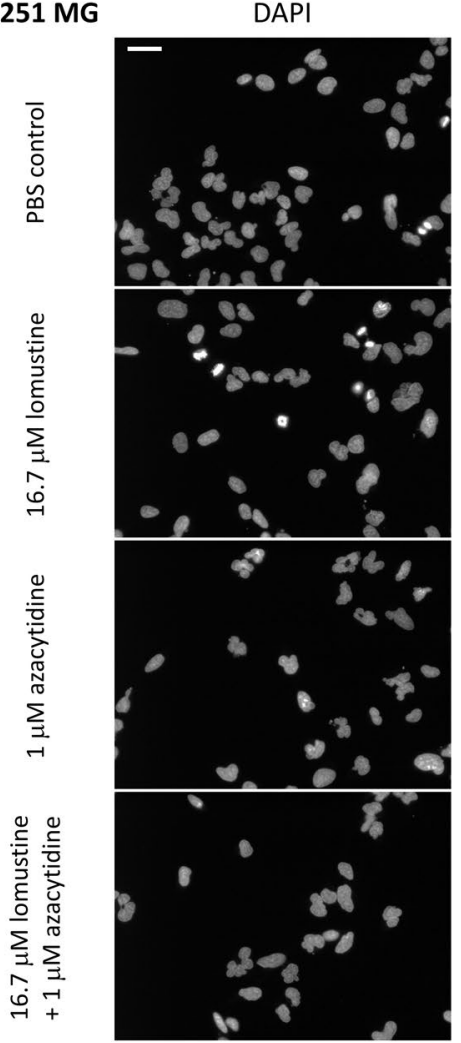

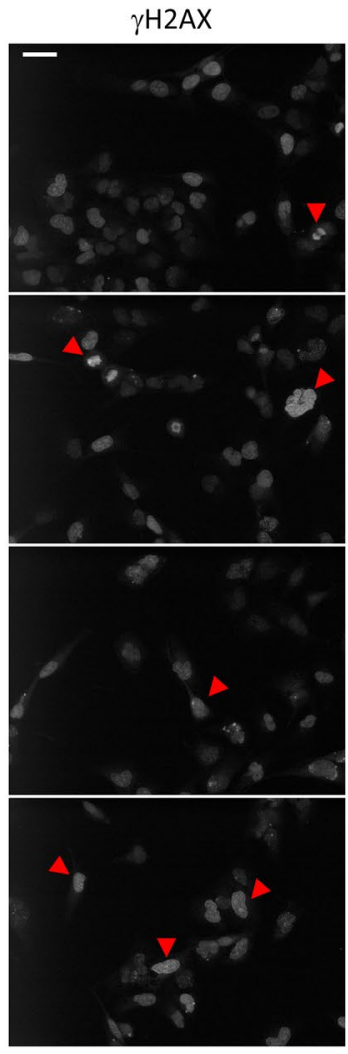

T98-G

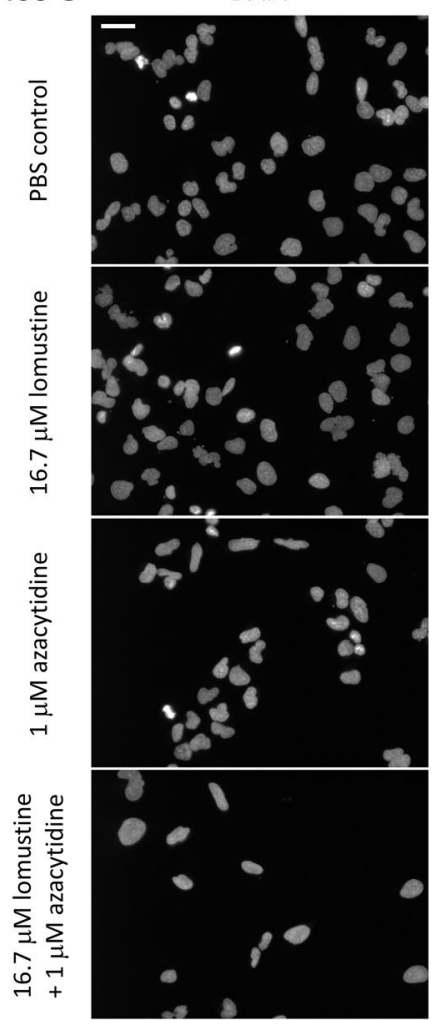

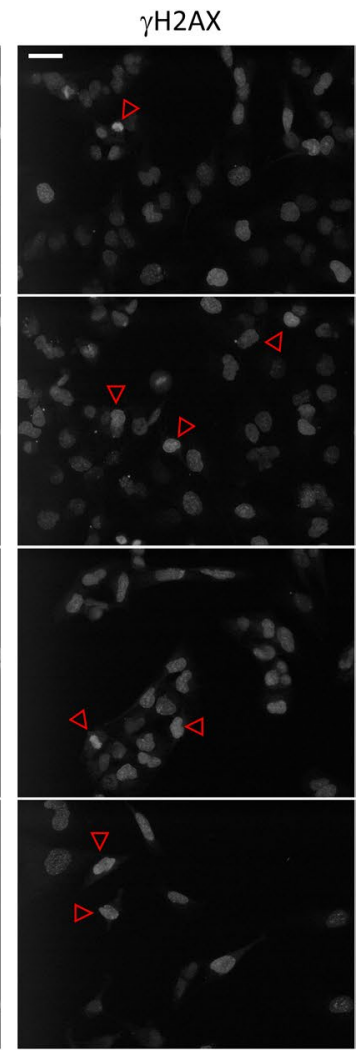

Figure 5. Representative microscopy images from IF assay in U-251 MG (left panel) and T98-G (right panel). The channels are indicated above the images and the treatment conditions ( $48 \mathrm{~h}$ ) on the left. For better visualization, the brightness of images was increased by $40 \%$ and the contrast reduced by $40 \%$; please note that the automated analysis of images was carried out using non-modified raw data. The images were collected within the same independent experiment. Scale bar (top left): $50 \mu \mathrm{m}$. Red arrowheads point to nuclei with strong $\gamma \mathrm{H} 2 \mathrm{AX}$ staining; empty arrowheads with red borders point to nuclei with medium levels of $\gamma \mathrm{H} 2 \mathrm{AX}$ staining.

lower than that of the $1: 1$ molar mixture $(P \leq 0.01)$, thus confirming that the mixture has higher efficiency than the individual compounds.

\section{Discussion}

Due to poor therapeutic outcome and limited available treatment strategies for recurring cases, GB remains a challenging disease. While new biologically active compounds are sought for GB treatment, the repurposing of the already available drugs in novel combinations also offers an advantageous alternative. From the set of compounds chosen for our study, several compounds have been previously characterized in the context of GB. Yet to our knowledge, there are no reports on the systematic in vitro studies that directly compare effects of selective compounds targeting crucial cellular pathways on the viability of GB cell lines-and which we hence undertook here.

Out of all tested targeted therapies, modulation of somatostatin receptors both with somatostatin receptor agonist and antagonist was the least effective. Although previous studies in C6 rat glioma xenografts have shown that simultaneous activation of different somatostatin receptors (SSTRs) inhibits tumor cell proliferation through both direct cytostatic and antiangiogenic effects, we did not see much efficacy in human GB cell lines ${ }^{35}$. The latter observation is in line with previous in vitro studies that confirmed that somatostatin peptides do not exert anti-proliferative effects in human GB cells and probably do not have obvious value for GB therapy ${ }^{36}$.

One of the cellular pathways that has gained increasingly much attention in the context of GB is PI3K/Akt/ mTOR. It has been shown that the gene encoding the phosphatase opposing activity of this pathway (PTEN) bears frequently inactivating mutations in $\mathrm{GB}^{37}$, thus necessitating the use of PI3K, Akt, or mTOR inhibitors to counteract the abnormally elevated activity of these kinases. The pan-Akt inhibitor MK-2206 was previously shown to enhance the effect of gefitinib in malignant glioma mouse model ${ }^{28}$, and act synergistically with temozolomide or irradiation in GB spheroids-yet not adherent cells ${ }^{29}$. However, these effects were consistently observed only at elevated concentrations of MK-2206 $(0.5-10 \mu \mathrm{M})$, which was somewhat unexpected given that this allosteric inhibitor does not have to compete with a high intracellular concentration of ATP. In our viability assay (Table 1), both MK-2206, as well as another allosteric pan-Akt inhibitor ARQ $092^{38,}$ showed one- to twodigit micromolar IC $_{50}$ values in U-251 MG and T98-G, respectively. Also, only a minimal increase in efficiency 
was observed upon combining ARQ 092 with lomustine (Fig. 2), which overall confirmed poor sensitivity of GB adherent cell lines to Akt inhibition.

Another potentially proto-oncogenic protein kinase that is abnormally active in GB is CK2, for which lowlevel amplifications were reported in one-third of glioblastomas, and which might also be indirectly connected to Akt signaling ${ }^{39}$. According to literature, the ATP-competitive inhibitor of CK2, CX-4945 suppressed the viability of GB cell lines (at $10 \mu \mathrm{M}$ or higher concentrations when used individually, or at $1 \mu \mathrm{M}$ concentration when combined with temozolomide), and was effective (individually or as a combination with temozolomide) in GB mouse models ${ }^{26,27,39}$. In our hands, the $\mathrm{IC}_{50}$ values for CX-4945 and its mixtures with temozolomide or lomustine also remained in the one- to two-digit micromolar range (Table 1, Fig. 2, Supplementary Figure S1), similarly to the $\mathrm{IC}_{50}$ values of the $\mathrm{CK} 2$ bisubstrate inhibitor ARC-775 (previously uncharacterized in GB context). It should however be noted that due to the limited solubility of CX-4945, its mixtures with chemotherapeutic agents contained high molar excess of the latter, thus improvement of solubility of CK2 inhibitors might be beneficial for more detailed studies of these compounds in the context of GB as well as other cancers.

Yet another family of protein kinases that is known to be aberrantly expressed in various cancers including GB is represented by the Aurora kinases, which are mapped on the intrinsically unstable regions of the genome $e^{40}$. Aurora A and B are indispensable for the progression of mitosis: Aurora A peaks at early mitosis, localizes to centrosomes and microtubules, and its overexpression overrides the mitotic checkpoint; Aurora B peaks at later phases of mitosis, and localizes to the kinetochores and midbody. Both kinases have been reported as the negative prognostic markers in $\mathrm{GB}^{41,42}$. Consistently, selective inhibitors of Aurora A [e.g., MLN8237 and VX 689923,30,41,43], Aurora B [e.g., AZD1152-HQPA ${ }^{21,22}$ ], as well as pan-Aurora inhibitors [e.g., AMG900, ZM 44743944,45] have been reported effective in GB cell lines as well as mouse models, when administered either as individual compounds, mixtures with temozolomide, or upon combination with irradiation.

In our hands, Aurora inhibitors, especially MLN8237 showed the highest efficiency among the tested individual compounds in both U-251 MG as well as T98-G (Table 1, Fig. 1); however, the viability of either cell line could not be reduced below $40 \%$ even at an elevated concentration of inhibitors, reflecting the fact that these compounds rather cause mitotic arrest than cell death per se. In mixtures of Aurora A inhibitors with chemotherapy agents, viability close to $0 \%$ was only observed at the highest concentration of temozolomide or lomustine, and fitting of dose-response curves indicated no improvement of IC $_{50}$ values for the mixtures relative to the individual Aurora inhibitors (Fig. 2, Supplementary Figure S1). This indicates that the potential administration of Aurora inhibitors as individual drugs or as mixtures with chemotherapy agents may not be sufficient to achieve therapeutic success in GB treatment. On the other hand, the arrest of cancerous cells in mitosis can be advantageous when combined with the DNA-damaging effect of ionizing radiation ${ }^{46}$. In our studies (Fig. 3), we confirmed that treatment with a relatively low concentration of MLN8237 (25 nM) either prior to or following irradiation of GB cells significantly enhanced the effect of radiation $(P \leq 0.01)$ even within the short timeframe of the experiment (the viability of cells was measured $48 \mathrm{~h}$ after irradiation). Therefore, a combination of inhibitors of Aurora kinases (and, possibly, other cell cycle-related targets) with radiation holds great potential for GB treatment and should be studied in detail regarding the dosages (i.e., a lower amount of inhibitor might be sufficient as compared to the studies exploring the effect of individual compounds) and the treatment schemes to achieve full potential.

Interestingly, inhibition of protein kinase Wee1, which is also involved in cell cycle regulation as well as DNA damage recognition and repair pathways, was in our case significantly more efficient in T98-G than in the U-251 MG cell line $(P \leq 0.001 ;$ Fig. 1$)$. In the case of Wee1 inhibitor MK-1775, earlier studies have reported the correlation of GB cell line resistance to the inhibitor with the elevated expression levels of Wee $1^{25}$. Contrarily, CC-115, the dual inhibitor of DNA-PK and mTOR, was significantly more efficient in U-251 MG than in the T98-G cell line $(P \leq 0.001$; Fig. 1$)$.

The two cell lines used for our study were chosen based on the reported differences in MGMT expression: U-251 MG was shown to have poorly detectable MGMT protein levels, whereas T98-G expressed MGMT ${ }^{47}$. While increased MGMT protein levels are associated with a poor prognosis for chemotherapeutic treatment in $\mathrm{GB}^{48,49}$, we did not observe major differences between temozolomide efficiency in viability assay in U-251 MG versus T98-G. We presume that there might be two major reasons. First, the differences in effects of alkylating agents have previously been reported for U-251 MG versus T98-G in viability assays with at least $72 \mathrm{~h}$ treatment times $^{50,51}$, or in the colony formation assays where the initial treatment times with compounds can be relatively short, yet the post-treatment period for monitoring recovery and growth of cells can last for weeks ${ }^{52}$. In our case, we performed viability assay directly following the treatment of cells, thus increasing the assay throughput, and enabling assessment of the initial, quick effects of compounds. Second, in our assay, temozolomide showed relatively high $\mathrm{IC}_{50}$ values in either cell line (Table 1); it is thus likely that in this range of $\mathrm{IC}_{50}$ values, the assay cannot distinguish sufficiently well between the subtle changes in cell viability following the short-term treatment. In case of lomustine as well as several other individual compounds, however, higher sensitivity of U-251 MG relative to T98-G could indeed be observed (Table 1, Fig. 1). Since the efficacy of cytotoxic chemotherapy and radiotherapy depends directly on DNA repair, the compounds and their combinations that augment the treatment-induced damage may also have a great potential in GB management. On the other hand, drug candidates that prove effective irrespective of the MGMT status of GB cells are also of utmost interest.

In the context of MGMT, the epigenetic regulation of gene expression have received a lot of attention in GB research: because methylation of the MGMT gene promoter is expected to reduce gene expression, the increased levels of MGMT promoter methylation are considered prognostic of the cancer responsiveness to treatment with cytotoxic cancer therapy ${ }^{48,49}$. Still, there has been conflicting evidence in the literature regarding the correlation between the MGMT methylation status and the enzyme expression level ${ }^{53,54}$. For instance, MGMT promoter methylation occurs in both U-251 MG and T98-G cell lines, whereas according to the enzyme expression level, U-251 MG is routinely classified as MGMT-negative and T98-G as MGMT-positive ${ }^{52}$. This is not surprising, 
as protein expression levels can be regulated via a variety of intracellular mechanisms, and the differences in ploidy of the cell lines of interest (diploid to triploid in case of U-251 MG, hyperpentaploid in case of T98-G) need also to be considered.

In our study, the epigenetic mechanisms were addressed via targeting DNA methyltransferases (DNMT) 1 and 3 (using azacytidine), and histone deacetylases (HDAC) 1 and 3 (using SAHA). Both classes of enzymes have been explored as drug targets in GB: while methylation of certain proteins can be beneficial for the survival of patients, hypermethylation of antiapoptotic genes can also occur ${ }^{55}$, and an imbalance between histone acyltransferases and HDACs has been reported for this disease ${ }^{56}$. The FDA-approved HDAC inhibitor SAHA has been studied in clinical trials for newly diagnosed GB (in combination with temozolomide or radiation) or recurrent $\mathrm{GB}^{18,19}$; in either case, the therapy had only modest efficacy. In our viability assays, SAHA also showed a very moderate, two-digit micromolar $\mathrm{IC}_{50}$ value-whether used individually (Table 1), or as a mixture with temozolomide or lomustine (Fig. 2, Supplementary Figure S1).

5 -Azacytidine, on the other hand, has been studied individually in glioma and GB xenografts ${ }^{16,17}$, and in patients with recurrent glioma bearing mutations of isocitrate dehydrogenase genes ${ }^{57}$, as the latter frequently exhibit DNA hypermethylation. Despite stratification of patients, no conclusive results regarding the efficiency of azacytidine monotherapy could be achieved ${ }^{57}$. In our viability assay with resazurin, $48 \mathrm{~h}$ treatment with azacytidine resulted in $8 \mu \mathrm{M}$ and $10 \mu \mathrm{M} \mathrm{IC}_{50}$ values in U-251 MG and T98-G, respectively (Table 1). Upon combining azacytidine with temozolomide or lomustine, the viability $\mathrm{IC}_{50}$ values were lowered fourfold in U-251 MG and threefold in T98-G relative to azacytidine alone; relative to lomustine alone, the 1:1 mixture of lomustine and azacytidine was over 20 -fold more potent (Fig. 2 and Supplementary Figure S2). Such enhancement of lomustine potency in both cell lines was remarkable, as we initially envisioned a risk of boosting U-251 MG resistance to lomustine upon inhibition of DNMT via possible increase in MGMT levels. Outside of the GB context, there are reports in the literature regarding the re-expression of MGMT upon application of azacytidine ${ }^{58-60}$. It is likely, however, that even if azacytidine-triggered demethylation results in an increase of MGMT expression, the net effect of the compound on cells will rather be dictated by the dramatic changes in the expression of multiple genes, including cell cycle regulators and proapoptotic factors such as CDKN2B, GADD45 $\gamma$ and FHIT ${ }^{61-63}$. Overall, this issue requires further detailed inspection in both in vitro and in vivo models of GB.

Within our current study, the gain in efficiency of lomustine + azacytidine mixtures as compared to the individual components was further confirmed using a different methodology. We developed a unique assay pipeline that utilizes automated imaging and machine learning-based data analysis algorithm for assessment of cell number following $48 \mathrm{~h}$ treatment and fixation of cells.

Machine learning approaches have become useful tools for cellular and biomedical image analysis and are used not only in academia but also for regular histological and pathological diagnostics including cancer diagnosis ${ }^{64,65}$. More specifically, numerous attempts have been made for nuclei detection from fluorescence images using various software solutions ${ }^{66-70}$. Although deep-learning techniques usually offer the highest quality in controlled conditions, it is possible that the once trained deep-learning networks lose some performance when introduced to imaging data from a different microscope, magnification and cell line (commonly known as the domain shift). Therefore, for solving tasks where a relatively low number of manual annotations and comparatively simpler machine learning solutions can provide sufficiently high-quality results, the choice of the machine learning tool comes down to usability, clarity of user interfaces and flexibility of integration with other analysis tools. As the Ilastik software ${ }^{71}$ fulfils these criteria, allows training models with sufficient quality for nuclei detection, requires no special expertise in machine learning and has good documentation, it was chosen as a basis for nuclei detection in this study. Although the Ilastik model in this study was specifically trained for detecting nuclei from GB cell lines, retraining or generating models for other specific cell lines or microscopy equipment can be done swiftly. In this case, we integrated Ilastik models with MembraneTools module of Aparecium software for image post-processing steps such as removal of too small objects, calculation of $\gamma \mathrm{H} 2 \mathrm{AX}$ channel intensity for each nucleus, and batch processing. However, depending on the need, batch processing can also be carried out directly in Ilastik or integrated with other bioimage analysis software, such as FIJI ${ }^{72}$, for post-processing.

According to the IF assay, even the mixture containing 17-fold molar excess of lomustine to azacytidine was significantly more potent $(P \leq 0.01)$ in reducing the number of $U-251 \mathrm{MG}$ and T98-G cells than the corresponding concentration of lomustine alone (Fig. 4a). In cells treated with lomustine + azacytidine mixtures, the $\gamma \mathrm{H} 2 \mathrm{AX}$ signal was not different from the signal observed for lomustine or azacytidine treatment alone (Fig. 4b), thus indicating that enhancement of lomustine potency by azacytidine occurs by other means than interference with DNA damage recognition or repair pathways.

The detailed study of cellular mechanisms affected by azacytidine and lomustine co-treatment will be addressed in our future studies. Furthermore, the knowledge regarding the delivery of azacytidine to the brain is currently limited, necessitating exhaustive planning of future experiments and clinical trials. Nevertheless, previous preclinical studies have confirmed that both intrathecal ${ }^{73}$ and intraperitoneal ${ }^{74}$ administration of 5 -azacytidine achieves adequate central nervous system exposure. Clinically, azacytidine is being tested in trials that allow participation of advanced solid tumor patients with treated and stable brain metastases ${ }^{75}$. Furthermore, taking into consideration that conventional radiotherapy can increase BBB permeability of chemotherapeutic $\operatorname{drugs}^{76}$, it is reasonable to believe that azacytidine reaches the brain tissue of glioblastoma patients-since almost all of them have received radiotherapy as their initial treatment, or as additional therapy in recurrent disease.

\section{Conclusions}

In corpora, these findings thus point to the high potential of combined therapy with lomustine and azacytidine in patients with recurrent GB, where the dosage of azacytidine can be tuned to avoid possible side-effects on bone marrow. Importantly, as both lomustine and azacytidine have been long used in clinical practice, there is a wealth 
of data regarding the safety of either drug. As our future goal, we thus aim to conduct an academic phase II clinical study to evaluate the safety and efficacy of the combination of DNMT1 inhibitor azacytidine and lomustine in recurrent glioblastoma patients, whose life expectancy with current treatments is approximately 7.8 months.

\section{Materials and methods}

Chemicals and equipment. Human glioblastoma astrocytoma cell line U-251 MG and human glioblastoma cell line T98-G were from the American Type Culture Collection (ATCC; Manassas, VA, USA). Temozolomide, lomustine, MLN8237 (alisertib), VX 689 (MK-5108), AZD1152-HQPA (barasertib), MK-1775 (adavosertib), CC-115 and ARQ 092 were obtained from Selleckchem (Munich, Germany). Pasireotide (trifluoroacetate salt), MK-2206, azacytidine and suberanilohydroxamic acid (SAHA) were from Cayman Chemical (Ann Arbor, Michigan, United States); CYN 154806 (trifluoroacetate salt) was from Tocris / Bio-Techne (Bristol, UK); CX-4945 (silmitasertib) was from Synkinase (Shanghai, China). ARC-775 (prepared and purified according to the previously published procedures ${ }^{34}$ ) was a kind gift of Dr Asko Uri (Institute of Chemistry, University of Tartu, Estonia). The stock solutions of compounds were prepared in cell culture grade DMSO was from AppliChem (Darmstadt, Germany) and stored at $-20^{\circ} \mathrm{C}$; stock solutions of pasireotide and CYN 154806 were aliquoted for single use to avoid repeated freeze-thawing. The chemicals and labware required for cell culturing, viability assay and fluorescence microscopy are listed under the Supplementary Methods.

Fluorescence intensity and absorbance measurements were carried out with Synergy NEO or Cytation 5 multi-mode readers (both from Biotek; Winooski, VT, USA). During the radiation studies, the cells were exposed to $6 \mathrm{MV}$ X-rays (Varian Truebeam 2.5). Fluorescence microscopy was carried out with Cytation 5 multi-mode reader using $20 \times$ air objective. For DAPI, $365 \mathrm{~nm}$ LED and DAPI filter block were used; for Alexa Fluor 568 , $523 \mathrm{~nm}$ LED and RFP filter block were used.

Treatment of cells for viability studies. The dose-response curves for individual compounds were performed in the 96-well format. U-251 MG or T98-G cells (passage number below 15) were seeded in growth medium onto the plate with a density of 3500 cells or 2500 cells per well, respectively (within the linear range of the method). After incubation for $24 \mathrm{~h}$, the growth medium was exchanged, and dilution series of biologically active compounds in PBS were added onto the cells (the final volume of PBS relative to the fetal bovine serumcontaining growth medium was $1 / 10$ ). Based on solubility of compounds in water, the following final total concentrations were chosen: temozolomide, lomustine, MK-1775, CC-115, azacytidine, SAHA, CYN 154806sixfold dilution starting from $100 \mu \mathrm{M}$; MK-2206, ARQ 092-sixfold dilution starting from $40 \mu \mathrm{M}$; pasireotide-sixfold dilution starting from $20 \mu \mathrm{M}$; ARC-775-sixfold dilution starting from $14 \mu \mathrm{M}$; MLN8237, VX 689, AZD1152-HQPA—sixfold dilution starting from $10 \mu \mathrm{M}$; CX-4945-sixfold dilution starting from 8.6 $\mu \mathrm{M}$. An identical volume of PBS was added to the negative control (100\% viability). The final volume per well was 100 $\mu \mathrm{L}$, and the concentration of DMSO in the treated wells was $\leq 0.1 \%$ by volume; on each plate, each concentration of each compound was represented in duplicate.

The dose-response curves for the mixtures of compounds with temozolomide or lomustine were performed analogously. For most compounds (MK1775, CC-115, azacytidine, SAHA, CYN 154806, AZD1152-HQPA), 1:1 stoichiometric ratio was used (relative to temozolomide or lomustine) for the dilution series. Other stoichiometric ratios used were as follows (the first number corresponds to the molar amount of temozolomide or lomustine, and the second number to the molar amount of added chemical in each well of the dilution series): MK-2206, ARQ 092-1:0.4; pasireotide-1:0.2; MLN8237, VX 689, AZD1152-HQPA-1:0.1; CX-4945-1:0.05. For Aurora A inhibitors, the dose-response curves of temozolomide or lomustine were additionally measured at the fixed concentration of the added chemical ( $1 \mathrm{nM}$ for MLN8237 and $10 \mathrm{nM}$ for VX 689).

The cells were incubated with individual compounds or mixtures for $48 \mathrm{~h}$, and viability assay was then carried out according to the previously published protocol ${ }^{77}$ (see details under the Supplementary Methods).

Radiation studies. The dose-response curves for individual compounds were performed in the 12 -well format. U-251 MG or T98-G cells (passage number below 15) were seeded in the growth medium onto plate with the density of 10,000 cells or 5000 cells per well, respectively (within the linear range of the method). After incubation for $24 \mathrm{~h}$, the growth medium was exchanged, and $25 \mathrm{nM}$ MLN8237 (diluted in the growth medium) was added to some wells ( 4 replicates per plate; pre-treatment). After another $24 \mathrm{~h}$, some plates were exposed to $2 \mathrm{~Gy}$ or $4 \mathrm{~Gy}$ of X-ray radiation; $2 \mathrm{~h}$ later, $25 \mathrm{nM}$ MLN8237 (diluted in the growth medium) was added to some wells on the irradiated or non-irradiated plates (4 replicates per plate; post-treatment). An identical volume of PBS was added to the negative control (100\% viability). The final volume per well was $1 \mathrm{~mL}$, and the concentration of DMSO in the treated wells was $0.1 \%$ by volume. Following the post-treatment, the cells were grown for another $48 \mathrm{~h}$, and viability assay was then carried out according to the previously published protocol ${ }^{77}$ (see details under the Supplementary Methods).

IF and fluorescence microscopy. The treatment of cells was carried out as described above, with adjustment for the working volume $(200 \mu \mathrm{L}$ per well $)$ and the number of seeded cells per well $(4,000$ cells in case of U-251 MG or 3000 cells in case of T98-G). Following $48 \mathrm{~h}$ treatment, the medium was removed, the cells were rinsed with PBS and fixed with cold methanol $\left(15 \mathrm{~min}\right.$ at $\left.-20^{\circ} \mathrm{C}\right)$. Next, the cells were washed with PBS and blocked for $1 \mathrm{~h}$ with $1 \%$ bovine serum albumin (BSA) in PBS $(\mathrm{w} / \mathrm{v})$ at room temperature; the overnight incubation at $4{ }^{\circ} \mathrm{C}$ with rabbit monoclonal IgG against $\gamma \mathrm{H} 2 \mathrm{AX}$ (Sigma-Aldrich, St Louis, MO, USA) followed (1:1000 dilution in $1 \% \mathrm{BSA} / \mathrm{PBS})$. Afterwards, the cells were washed with $0.1 \%$ solution of Triton X-100 in PBS, and 3-6 h incubation at $4{ }^{\circ} \mathrm{C}$ with goat cross-adsorbed antibody against rabbit $\operatorname{IgG}(\mathrm{H}+\mathrm{L})$ conjugated with Alexa Fluor 568 (Invitrogen; Eugene, OR, USA) was carried out (1:1000 dilution in 1\% BSA/PBS). The washing pro- 
cedure was repeated, followed by 5 min staining with $300 \mathrm{nM}$ solution of the nuclear stain DAPI (Invitrogen, Eugene, OR, USA) in PBS. Finally, the cells were washed with PBS and stored in PBS at $4{ }^{\circ} \mathrm{C}$ until imaging.

The imaging was performed in the automated mode; 25 images per well were taken and DAPI channel was used for autofocusing. The imaging settings (LED intensity/signal integration time/detector gain) were as follows: for DAPI, 4/104/5; for Alexa Fluor ${ }^{\circledR} 568,6 / 252 / 19$. The examples of strong nuclear staining indicating the suitability of the $\gamma \mathrm{H} 2 \mathrm{AX}$ antibody for the assay are presented in Supplementary Figure S7.

Automated image analysis. For automated image analysis, a total of 11 images from DAPI channel were chosen for the training set containing images from multiple sequential focal planes to increase the algorithmic robustness against minor focusing errors. Ilastik software version 1.3.3b2 was used for all annotations and model training ${ }^{71}$. In the model, six features were used: Gaussian smoothing ( $\sigma_{0}$ value of 0.3 pixels), Laplacian of Gaussian, Gaussian gradient magnitude, difference of Gaussians, structure tensor eigenvalues and Hessian of Gaussian eigenvalues (all the features utilized $\sigma_{1-8}$ values of $0.7,1,1.6,3.5,5,10,20$ and 35 pixels). The pixels were classified into either the nuclei or background classes and all annotations were performed in Live Update mode. Model quality was incrementally increased by adding additional annotations to areas where the pixel misclassification was the highest. In most cases, these areas were located near the nucleus edge. The classification probability maps were binarized by using the probability threshold of 0.5 . Nuclei with an area smaller than 200 pixels $(20.8$ $\mu \mathrm{m}^{2}$ ) were removed, as such objects were considered to be imaging or fixation artifacts. The trained model is available at https://gpcr.ut.ee/aparecium.html. The pipeline robustness against detection of possible microscopy artifacts was checked and confirmed visually. Quality metrics for the algorithm (see details in the Supplementary Table S2) were determined to be as follows: F1 score $=0.94$, precision $=0.91$, recall $=0.97$, Matthews correlation coefficient $(\mathrm{MCC})=0.96$. All quality metrics were thus sufficiently close to the perfect detection indicating that the image analysis pipeline was fit for purpose. Batch processing was carried out using the modified version of the MembraneTools module of Aparecium 2.0 software $^{78}$. The software is available at https:/gpcr.ut.ee/apare cium.html. Based on the location of the nuclear pixels, the objects were segmented into individual nuclei by connected component analysis. The average and total intensities of the individual nuclei were calculated for each image in both DAPI and $\gamma \mathrm{H} 2 \mathrm{AX}$ imaging channels. The data with average intensities were exported to commaseparated values (CSV) format and used for further statistical analysis.

Statistical analysis. For data analysis, GraphPad Prism 6 (San Diego, CA, USA) and Excel 2016 (Microsoft Office 365; Redmond, WA, USA) were used. In the case of all methods, at least three independent experiments were performed. Throughout the study, the grouped comparisons were carried out using 1-way ANOVA with Dunnett's test for multiple comparisons; unless indicated otherwise, the pairwise comparisons were carried out using the unpaired two-tailed t-test with Welch's correction. In all statistical tests, the significance of comparisons is indicated as follows: ${ }^{* *}$ indicates $P \leq 0.001,{ }^{* *}$ indicates $P \leq 0.01,{ }^{*}$ indicates $P \leq 0.05$. The details on the normalization of data and statistical analyses are provided under the Supplementary Methods.

\section{Data availability}

The datasets generated and analyzed during the current study are available from the corresponding author on reasonable request. The software and Ilastik model is available at https://doi.org/10.6084/m9.figshare.14749530.v1. The examples of non-modified microscopy images are available at https://figshare.com/s/35c7b3a19270592f881f.

Received: 3 August 2021; Accepted: 24 September 2021

Published online: 13 October 2021

\section{References}

1. Crocetti, E. et al. Epidemiology of glial and non-glial brain tumours in Europe. Eur. J. Cancer 48, 1532-1542 (2012).

2. Walker, M. D. et al. Evaluation of BCNU and/or radiotherapy in the treatment of anaplastic gliomas: A cooperative clinical trial. J. Neurosurg. 49, 333-343 (1978).

3. Stupp, R. et al. Radiotherapy plus concomitant and adjuvant temozolomide for glioblastoma. N. Engl. J. Med. 352, 987-996 (2005).

4. Stupp, R. et al. Effect of tumor-treating fields plus maintenance temozolomide vs maintenance temozolomide alone on survival in patients with glioblastoma: A randomized clinical trial. JAMA 318, 2306 (2017).

5. Wick, W. et al. Phase III study of enzastaurin compared with lomustine in the treatment of recurrent intracranial glioblastoma. $J$. Clin. Oncol. 28, 1168-1174 (2010).

6. Batchelor, T. T. et al. Phase III randomized trial comparing the efficacy of cediranib as monotherapy, and in combination with lomustine, versus lomustine alone in patients with recurrent glioblastoma. J. Clin. Oncol. 31, 3212-3218 (2013).

7. Taal, W. et al. Single-agent bevacizumab or lomustine versus a combination of bevacizumab plus lomustine in patients with recurrent glioblastoma (BELOB trial): A randomised controlled phase 2 trial. Lancet Oncol. 15, 943-953 (2014).

8. Brandes, A. A. et al. A Phase II randomized study of galunisertib monotherapy or galunisertib plus lomustine compared with lomustine monotherapy in patients with recurrent glioblastoma. Neuro Oncol. 18, 1146-1156 (2016).

9. Lombardi, G. et al. Regorafenib compared with lomustine in patients with relapsed glioblastoma (REGOMA): A multicentre, open-label, randomised, controlled, phase 2 trial. Lancet Oncol. 20, 110-119 (2019).

10. Kazmi, F., Soon, Y. Y., Leong, Y. H., Koh, W. Y. \& Vellayappan, B. Re-irradiation for recurrent glioblastoma (GBM): A systematic review and meta-analysis. J. Neurooncol. 142, 79-90 (2019).

11. Di Cintio, F. et al. The molecular and microenvironmental landscape of glioblastomas: Implications for the novel treatment choices. Front. Neurosci. 14 (2020).

12. Birzu, C. et al. Recurrent glioblastoma: From molecular landscape to new treatment perspectives. Cancers 13, 47 (2021).

13. Khaddour, K., Johanns, T. \& Ansstas, G. The landscape of novel therapeutics and challenges in glioblastoma multiforme: Contemporary state and future directions. Pharmaceuticals 13 (2020).

14. Barazzuol, L. et al. Evaluation of poly (ADP-ribose) polymerase inhibitor ABT-888 combined with radiotherapy and temozolomide in glioblastoma. Radiat. Oncol. 8, 65 (2013). 
15. Mansouri, A. et al. MGMT promoter methylation status testing to guide therapy for glioblastoma: Refining the approach based on emerging evidence and current challenges. Neuro Oncol. 21, 167-178 (2019).

16. Borodovsky, A. et al. 5-azacytidine reduces methylation, promotes differentiation and induces tumor regression in a patient-derived IDH1 mutant glioma xenograft. Oncotarget 4, 1737-1747 (2013).

17. Kratzsch, T. Treatment with 5-azacitidine delay growth of glioblastoma xenografts: A potential new treatment approach for glioblastomas. J. Cancer Res. Clin. Oncol. 144, 809-819 (2018).

18. Galanis, E. et al. Phase II trial of vorinostat in recurrent glioblastoma multiforme: A north central cancer treatment group study. JCO 27, 2052-2058 (2009).

19. Galanis, E. et al. Phase I/II trial of vorinostat combined with temozolomide and radiation therapy for newly diagnosed glioblastoma: Results of Alliance N0874/ABTC 02. Neuro Oncol. 20, 546-556 (2018).

20. Ceccato, F. et al. Temozolomide and pasireotide treatment for aggressive pituitary adenoma: Expertise at a tertiary care center. J. Neurooncol. 122, 189-196 (2015).

21. Zekri, A. et al. Inhibitor of aurora Kinase B induces differentially cell death and polyploidy via DNA damage response pathways in neurological malignancy: Shedding new light on the challenge of resistance to AZD1152-HQPA. Mol. Neurobiol. 53, 1808-1823 (2016).

22. Diaz, R. J. et al. Mechanism of action and therapeutic efficacy of Aurora kinase. Oncotarget 6, 3360-3374 (2014).

23. Bjerke, L. et al. Histone H3.3 mutations drive paediatric glioblastoma through upregulation of MYCN. Cancer Discov. 3, 512-519 (2014).

24. Sanai, N. et al. Phase 0 trial of AZD1775 in first-recurrence glioblastoma patients. Clin. Cancer Res. 24, 3820-3828 (2018).

25. Pokorny, J. L. et al. The efficacy of the Wee1 inhibitor MK- 1775 combined with temozolomide is limited by heterogeneous distribution across the blood-brain barrier in glioblastoma. Clin. Cancer Res. 21, 1916-1924 (2015).

26. Liu, X., Chen, J., Li, W., Hang, C. \& Dai, Y. Inhibition of casein kinase II by CX-4945, but not yes-associated protein (YAP) by verteporfin, enhances the antitumor efficacy of temozolomide in glioblastoma. Transl. Oncol. 13, 70-78 (2020).

27. Ferrer-Font, L. et al. Targeting protein kinase CK2: Evaluating CX-4945 potential for GL261 glioblastoma therapy in immunocompetent mice. Pharmaceuticals 10 (2017).

28. Cheng, Y. et al. MK-2206, a novel allosteric inhibitor of Akt, synergizes with Gefitinib against malignant glioma via modulating both autophagy and apoptosis. Mol. Cancer Therap. 11, (2012).

29. Narayan, R. S. The allosteric AKT inhibitor MK2206 shows a synergistic interaction with chemotherapy and radiotherapy in glioblastoma spheroid cultures. BMC Cancer 17, 204 (2017).

30. Hong, X. et al. The selective Aurora-A kinase inhibitor MLN8237 (alisertib) potently inhibits proliferation of glioblastoma neurosphere tumor stem-like cells and potentiates the effects of temozolomide and ionizing radiation. Cancer Chemother. Pharmacol. 73, 983-990 (2014).

31. Munster, P. et al. First-in-human phase I study of a dual mTOR kinase and DNA-PK inhibitor (CC-115) in advanced malignancy. Cancer Manag. Res. 11, 10463-10476 (2019).

32. Nunn, C., Schoeffter, P., Langenegger, D. \& Hoyer, D. Functional characterisation of the putative somatostatin sst2 receptor antagonist CYN 154806. Naunyn-Schmiedeberg's Arch. Pharmacol. 367, 1-9 (2003).

33. Feniuk, W., Jarvie, E., Luo, J. \& Humphrey, P. P. A. Selective somatostatin sst2 receptor blockade with the novel cyclic octapeptide, CYN-154806. Neuropharmacology 39, 1443-1450 (2000).

34. Rahnel, H. et al. A selective biligand inhibitor of CK2 increases caspase-3 activity in cancer cells and inhibits platelet aggregation. ChemMedChem 12, 1723-1736 (2017).

35. Barbieri, F. et al. Differential efficacy of SSTR1, -2, and -5 agonists in the inhibition of C6 glioma growth in nude mice. Am. J. Physiol, Endocrinol. Metab. 297, E1078-E1088 (2009).

36. Lamszus, K., Meyerhof, W. \& Westphal, M. Somatostatin and somatostatin receptors in the diagnosis and treatment of gliomas. J. Neurooncol. 35, 353-364 (1997).

37. Knobbe, C. B. \& Reifenberger, G. Genetic alterations and aberrant expression of genes related to the phosphatidyl-lnositol-3'kinase/protein kinase B (Akt) signal transduction pathway in glioblastomas. Brain Pathol. 13, 507-518 (2003).

38. Yu, Y. et al. Targeting AKT1-E17K and the PI3K/AKT pathway with an allosteric AKT inhibitor, ARQ 092. PLoS ONE 10, e0140479 (2015).

39. Zheng, Y. et al. Targeting protein kinase CK2 suppresses prosurvival signaling pathways and growth of glioblastoma. Clin. Cancer Res. 19, 6484-6494 (2013).

40. Tang, A. et al. Aurora kinases: Novel therapy targets in cancers. Oncotarget 8, 23937-23954 (2017).

41. Lehman, N. L. et al. Aurora A is differentially expressed in gliomas, is associated with patient survival in glioblastoma and is a potential chemotherapeutic target in gliomas. Cell Cycle 11, 489-502 (2012).

42. Zeng, W. F., Navaratne, K., Prayson, R. A. \& Weil, R. J. Aurora B expression correlates with aggressive behaviour in glioblastoma multiforme. J. Clin. Pathol. 60, 218-221 (2007).

43. Willems, E. et al. Aurora A plays a dual role in migration and survival of human glioblastoma cells according to the CXCL12 concentration. Oncogene 38, 73-87 (2019).

44. Ryu, J., Pyo, J., Lee, C. \& Kim, J. An Aurora kinase inhibitor, AMG900, inhibits glioblastoma cell proliferation by disrupting mitotic progression. Cancer Med. 7, 5589-5603 (2018).

45. Borges, K. S. et al. Inhibition of Aurora kinases enhances chemosensitivity to temozolomide and causes radiosensitization in glioblastoma cells. J. Cancer Res. Clin. Oncol. 138, 405-414 (2012).

46. Biau, J., Chautard, E., Verrelle, P. \& Dutreix, M. Altering DNA repair to improve radiation therapy: Specific and multiple pathway targeting. Front. Oncol. 9 (2019).

47. Chahal, M. et al. O(6)-Methylguanine-DNA methyltransferase is a novel negative effector of invasion in glioblastoma multiforme. Mol. Cancer Ther. 11, 2440-2450 (2012).

48. Esteller, M. et al. Inactivation of the DNA-repair gene MGMT and the clinical response of gliomas to alkylating agents. N. Engl. J. Med. 343, 1350-1354 (2000).

49. Wick, W. et al. Lomustine and Bevacizumab in progressive glioblastoma. N. Engl. J. Med. 377, 1954-1963 (2017).

50. Natsume, A. et al. IFN- $\beta$ down-regulates the expression of DNA repair gene MGMT and sensitizes resistant glioma cells to temozolomide. Cancer Res 65, 7573-7579 (2005).

51. Ryu, C. H. et al. Valproic acid downregulates the expression of MGMT and sensitizes temozolomide-resistant glioma cells. J. Biomed. Biotechnol. 2012, 987495 (2012).

52. van Nifterik, K. A. et al. Absence of the MGMT protein as well as methylation of the MGMT promoter predict the sensitivity for temozolomide. Br. J. Cancer 103, 29-35 (2010).

53. Kreth, S. et al. O6-Methylguanine-DNA methyltransferase (MGMT) mRNA expression predicts outcome in malignant glioma independent of MGMT promoter methylation. PLOS ONE 6, e17156 (2011).

54. Pandith, A. A. et al. Concordant association validates MGMT methylation and protein expression as favorable prognostic factors in glioma patients on alkylating chemotherapy (Temozolomide). Sci. Rep. 8, 6704 (2018).

55. Li, P. \& Wu, M. Epigenetic Mechanisms of Glioblastoma. in Glioblastoma (ed. De Vleeschouwer, S.) (Codon Publications, 2017).

56. Chen, R. et al. The application of histone deacetylases inhibitors in glioblastoma. J. Exp. Clin. Cancer Res 39 (2020).

57. Federici, L. et al. 5-Azacitidine in patients with IDH1/2-mutant recurrent glioma. Neuro Oncol. 22, 1226-1228 (2020). 
58. Su, Y. et al. Azacytidine inhibits the proliferation of human promyelocytic leukemia cells (HL60) by demethylation of MGMT, DAPK and p16 genes. Hematology 17, 41-46 (2012).

59. Ren, X., Li, H., Song, X., Wu, Y. \& Liu, Y. 5-Azacytidine treatment induces demethylation of DAPK1 and MGMT genes and inhibits growth in canine mammary gland tumor cells. Onco Targets Ther. 11, 2805-2813 (2018).

60. Yamada, H., Vijayachandra, K., Penner, C. \& Glick, A. Increased sensitivity of transforming growth factor (TGF) $\beta 1$ null cells to alkylating agents reveals a novel link between TGF $\beta$ signaling and O 6-methylguanine methyltransferase promoter hypermethylation*. J. Biol. Chem. 276, 19052-19058 (2001).

61. Kimura, S. et al. Antiproliferative and antitumor effects of azacitidine against the human myelodysplastic syndrome cell line SKMAnticancer Res. 32, 795-798 (2012).

62. Tran, H. T. T. et al. DNA methylation changes following 5-azacitidine treatment in patients with myelodysplastic syndrome. J. Korean Med. Sci. 26, 207 (2011).

63. Yang, Y., Li, J., Geng, Y., Liu, L. \& Li, D. Azacitidine regulates DNA methylation of GADD45 $\gamma$ in myelodysplastic syndromes. J. Clin. Lab Anal. 35, e23597 (2020).

64. Angermueller, C., Pärnamaa, T., Parts, L. \& Stegle, O. Deep learning for computational biology. Mol. Syst. Biol. 12, 878 (2016).

65. Bankhead, P. et al. QuPath: Open source software for digital pathology image analysis. Sci. Rep. 7, 16878 (2017).

66. Carpenter, A. E. et al. Cell Profiler: Image analysis software for identifying and quantifying cell phenotypes. Genome Biol. 7, R100 (2006).

67. Coelho, L. P., Shariff, A. \& Murphy, R. F. Nuclear segmentation in microscope cell images: A hand-segmented dataset and comparison of algorithms. Proc. IEEE Int. Symp. Biomed. Imaging 5193098, 518-521 (2009).

68. Xing, F. \& Yang, L. Robust nucleus/cell detection and segmentation in digital pathology and microscopy images: A comprehensive review. IEEE Rev. Biomed. Eng. 9, 234-263 (2016).

69. Li, G. et al. Segmentation of touching cell nuclei using gradient flow tracking. J. Microsc. 231, 47-58 (2008).

70. Salvi, M. et al. Automated segmentation of fluorescence microscopy images for 3D cell detection in human-derived cardiospheres. Sci. Rep. 9, 6644 (2019).

71. Berg, S. et al. ilastik: Interactive machine learning for (bio)image analysis. Nat. Methods 16, 1226-1232 (2019).

72. Schindelin, J. et al. Fiji: An open-source platform for biological-image analysis. Nat. Methods 9, 676-682 (2012).

73. Lester McCully, C. et al. Plasma and cerebrospinal fluid pharmacokinetics of the DNA methyltransferase inhibitor, 5-azacytidine, alone and with inulin, in nonhuman primate models. Neuro-Oncol. Adv. 2 (2020).

74. Butler, C. et al. Hypomethylating agent azacitidine is effective in treating brain metastasis triple-negative breast cancer through regulation of DNA methylation of keratin 18 gene. Transl. Oncol. 13, 100775 (2020).

75. Cheng, Y. et al. Targeting epigenetic regulators for cancer therapy: Mechanisms and advances in clinical trials. Sig. Transduct. Target Ther. 4, 1-39 (2019).

76. Luo, H. \& Shusta, E. V. Blood-brain barrier modulation to improve glioma drug delivery. Pharmaceutics 12, 1085 (2020).

77. Lavogina, D. et al. Chemosensitivity and chemoresistance in endometriosis-Differences for ectopic versus eutopic cells. Reprod. Biomed. Online 39, 556-568 (2019).

78. Allikalt, A., Laasfeld, T., Ilisson, M., Kopanchuk, S. \& Rinken, A. Quantitative analysis of fluorescent ligand binding to dopamine D3 receptors using live-cell microscopy. FEBS J. 288, 1514-1532 (2021).

\section{Acknowledgements}

We thank Ago Rinken for providing access to the microplate reader facility and Asko Uri for the CK2 inhibitor ARC-775.

\section{Author contributions}

H.L. was responsible for culturing and treatment of cells; M.V. was responsible for radiation experiments; T.L. developed and validated the automated image analysis algorithm; D.L. conducted viability assay, immunostaining and fluorescence microscopy, performed data analysis, and drafted the manuscript; D.L., M.V. and J.J. were involved in conception and design of the study and performed critical revision of the manuscript. All authors read and approved the final version of the manuscript.

\section{Funding}

The study was supported by the internal financing from the Institute of Clinical Medicine, University of Tartu, Estonia (2019-2022), and by the Estonian Ministry of Education and Research (PRG454).

\section{Competing interests}

The authors declare no competing interests.

\section{Additional information}

Supplementary Information The online version contains supplementary material available at https://doi.org/ 10.1038/s41598-021-99630-0.

Correspondence and requests for materials should be addressed to D.L. or J.J.

Reprints and permissions information is available at www.nature.com/reprints.

Publisher's note Springer Nature remains neutral with regard to jurisdictional claims in published maps and institutional affiliations. 
(c) (i) Open Access This article is licensed under a Creative Commons Attribution 4.0 International cc) License, which permits use, sharing, adaptation, distribution and reproduction in any medium or format, as long as you give appropriate credit to the original author(s) and the source, provide a link to the Creative Commons licence, and indicate if changes were made. The images or other third party material in this article are included in the article's Creative Commons licence, unless indicated otherwise in a credit line to the material. If material is not included in the article's Creative Commons licence and your intended use is not permitted by statutory regulation or exceeds the permitted use, you will need to obtain permission directly from the copyright holder. To view a copy of this licence, visit http://creativecommons.org/licenses/by/4.0/.

(C) The Author(s) 2021 